\title{
Guest Editorial: Functional Somatic Syndromes
}

\author{
Urs M. Nater
}

Published online: 17 April 2013

(C) International Society of Behavioral Medicine 2013

\section{Introduction}

Somatic symptoms that cannot be readily explained by modern medicine are labeled as "functional" or "medically unexplained," i.e., after thorough medical examination, no structural pathology and no proportional tissue abnormalities can be identified for these symptoms. Functional somatic syndromes (FSS) are defined by a constellation of functional or medically unexplained symptoms [1]. Among the most prominent and prevalent FSS are chronic fatigue syndrome, irritable bowel syndrome, and fibromyalgia syndrome. But there are many more: chronic pelvic pain, low back pain, tension headache, noncard iac chest pain, multiple chemical sensitivity, the sick building syndrome, chronic whiplash syndrome, the Gulf War syndrome, and temporomandibular joint syndrome, just to name a few. Although a literature search on PubMed using the term "functional somatic syndromes" yields almost 1,000 hits (and there are many more when searching for single FSS, such as chronic fatigue syndrome), it is remarkable how little is known about those enigmatic conditions. For example, it is still a major challenge in the field of FSS (and medicine in general) to develop concepts of what "no structural pathology and no proportional tissue abnormalities" actually means; maybe modern medicine is just not yet adequately equipped with the technology needed in order to detect abnormalities that may fully explain symptoms of FSS? Maybe it is just a matter of time until a common medical explanation can be found for each FSS. One instantly thinks of "stress-related ulcers," which had been thought of as expressions of an underlying psychosomatic process - but were ultimately found to be caused by a bacterium called Helicobacter

U. M. Nater $(\bowtie)$

Department of Psychology, University of Marburg,

Gutenbergstrasse 18,

35032 Marburg, Germany

e-mail: nater@uni-marburg.de pylori. Although this finding does not exclude an interaction of psychological and biological processes in the manifestation of ulcers, it clearly shows that the matter of "medical explanation" is challenging. It is a very pragmatic question that asks whether it is at all relevant to distinguish between medically explained and medically unexplained symptoms, then. In the first paper of this special series, Klaus et al. convincingly argue that, from a clinical perspective, it is not relevant: both medically explained and medically unexplained symptoms result in comparable impairment and persist equally over time. A similar approach will be taken in the new edition of the classification manual of psychiatric conditions, the Diagnostic and Statistical Manual of Mental Disorders- $V$, in which the previously used term "somatoform," i.e., medically unexplained, is going to be abandoned, mostly due to the reasoning that it does not matter clinically whether bodily symptoms may be medically explained or not, but whether symptoms are causing distress or not, and how patients are dealing with their symptoms.

Another point of consideration deals with the question whether FSS are distinct syndromes, independent from each other, or whether there is a common underlying process responsible for the manifestation of FSS symptoms. In the latter case, it may be just happenstance or depending on medical specialization of the diagnostician what combination of symptoms may ultimately lead to what FSS diagnosis. A number of studies have shown that it might indeed be the case that FSS may not be separate from others, but that there might be one unifying syndrome which encompasses all of the (e.g., above-mentioned) FSS. The jury is still out on this, but two studies in the current special series indicate that it is not a simple one or the other: Witthöft et al. show that there is a common underlying factor of medically unexplained symptoms, thus hinting at the notion that there might be a single large category of functional somatic illness; however, the authors also found that there were four symptom-specific factors in addition to this general factor, 
which they were able to relate to specific FSS. This "bifactor model," as the authors call it, may be an important addition to the discussion of whether there is one or many FSS. The other paper dealing with this question focuses on prevalence and overlap of FSS: Fischer et al. show that, while prevalence of FSS is high even in a non-clinical sample, only a few participants actually fulfill the diagnostic criteria for more than one FSS. In contrast to previous studies, this may be a matter of how diagnosis was approached: in this study, exclusionary criteria and possible explanations by a doctor were assessed, a factor that was neglected in many other studies, in which merely "positive" criteria were assessed (i.e., the presence of diagnostic symptoms, but not "proof" of the absence of potentially exclusionary explanations).

A final set of papers deals with potential predictors of FSS. As initially mentioned, it is surprising how few common factors have been identified which have the potential to explain etiology and maintenance of FSS. A case could be made that conceptual issues may forever prevent one from reaching final conclusions regarding pathophysiology, but such a defeatist notion is probably not helpful at this point. Some of the papers in this special series have addressed separate aspects of potential predictors: Creed et al. suggest that the presence of multiple somatic symptoms may predict poor health in selected FSS. As these findings are derived from a longitudinal study, it may be of critical relevance to implement the assessment of multiple somatic symptoms in order to reach clinically significant treatment decisions. Another avenue of research has focused on the role of psychological factors which might, at least in part, explain the presence of FSS. In their article, Kingma et al. found that (similar to the previously mentioned study) multiple symptoms, as well as female gender and, interestingly, high intelligence, were predictors of receiving a diagnosis of FSS. Whether intelligence per se is an etiological factor or whether this trait might increase the possibility that patients seek help or are labeled as having an FSS remains to be determined. Another important factor that has been examined was symptom expectancies; the role of dysfunctional cognitions in the development of FSS has been shown repeatedly. In their study, Heins et al. focused on maladaptive expectancies regarding stair climbing in patients with chronic fatigue syndrome (CFS). They were able to show that such anticipatory cognitions have a direct consequence on daily activities in these patients. It is not a coincidence that this study was conducted in the context of CFS: in fact, CFS remains the best-studied FSS. At the same time, it also remains one that is not very well understood. The final two papers of the special series focus on psychological distress in CFS, which is of great relevance considering the burden weighing on suffering patients. Kempke et al. show in their study that depressive and obsessive-compulsive traits are associated with CFS, a finding that is mirrored by the study of Marques et al., in which the relevance of anxiety and depression are highlighted. Clearly, more research is needed on the role of psychological factors, particularly regarding the question of whether psychological abnormalities might be the consequence of suffering from a disabling illness or whether they might lay the ground for later development of FSS.

Thus, the papers in this special series both corroborate known facts and provide new insights in FSS, certainly reflecting the current state of research in FSS. This is very succinctly summarized in the comment by Harvey and Wessely at the end of this special series, where future directions are further delineated. Clearly, research on FSS remains profoundly relevant and clinically meaningful, and with this special series, it is our sincere hope that both researchers and clinicians will find sufficient information that may guide both their research and treatment decisions.

\section{Reference}

1. Barsky AJ, Borus JF. Functional somatic syndromes. Ann Intern Med. 1999;130(11):910-21. 